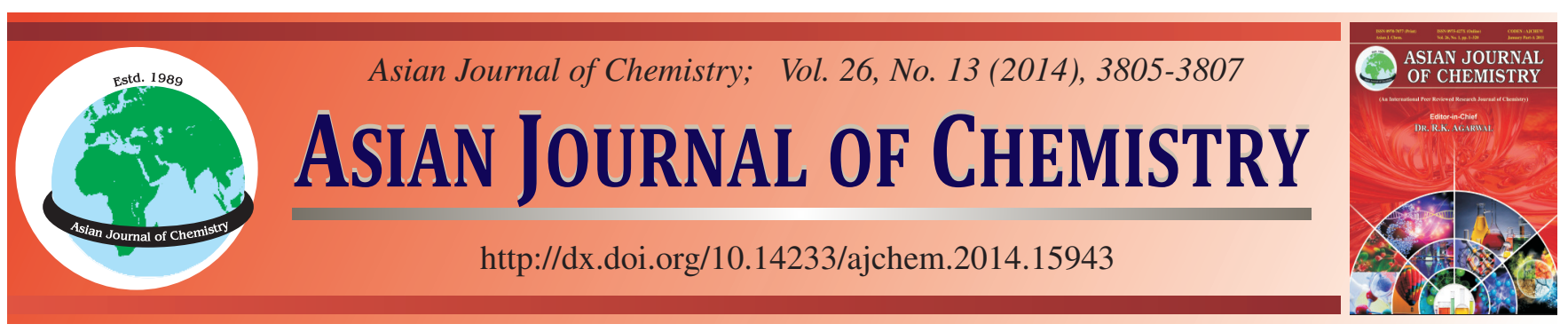

\title{
Antibacterial Activity of Naphthyridone Derivatives Containing 8-Alkoxyimino-1,6-dizaspiro[3,4]octane Scaffolds
}

\author{
Lianshun Feng ${ }^{1,2,3, \dagger}$, Yanhong Tan $^{2, \dagger}$, Zengquan Wei $^{1}$, Mingliang Liu ${ }^{1, *}$ and Huiyuan Guo ${ }^{1}$
}

${ }^{1}$ Institute of Medicinal Biotechnology, Chinese Academy of Medical Sciences and Peking Union Medical College, Beijing 100050, P.R. China ${ }^{2}$ Beijing National Laboratory for Molecular Sciences, Department of Chemical Biology, College of Chemistry and Molecular Engineering, Peking University, Beijing 100871, P.R. China

${ }^{3}$ Hybio Pharmaceutical Co., Ltd, Shenzhen 518057, P.R. China

*Corresponding author: Tel/Fax: +86 10 63036965; E-mail: 1mllyx@126.com

$\dagger$ These authors contributed equally to the work

We report herein in vitro antibacterial activity of a series of novel naphthyridone derivatives containing 8-alkoxyimino-1,6-dizaspiro[3.4] octane scaffolds, the position isomers of the side chain at the C-7 position of zabofloxacin. Our results revealed that the target compounds were generally less active than the reference against the tested Gram-positive and Gram-negative strains with few exceptions, but compounds 1-5 and 7 showed good activity against MSSA and P. aeruginosa (MICs: $0.25-1 \mu \mathrm{g} / \mathrm{mL}$ ). Especially, compounds 3 and 7 (MICs: $0.25 \mu \mathrm{g} / \mathrm{mL}$ ) were found to be 4 times more potent than or comparable to levofloxacin against $P$. aeruginosa, the important conditioned pathogen on hospital infection. Simple structure-activity relationship was also discussed in this paper.

Keywords: Naphthyridone derivatives, 8-Alkoxyimino-1,6-dizaspiro[3.4]octane Scaffolds, Antibacterial activity.

\section{INTRODUCTION}

Quinolones, exert their effect by inhibition of two type II bacterial topoisomerase enzymes, DNA gyrase (topoisomerase II) and topoisomerase IV $^{1,2}$, have been used to treat various diseases including respiratory tract infections, urinary tract infections, sexually transmitted diseases, gastrointestinal and abdominal infections, skin and soft tissue infections and infections of the bone and joints ${ }^{3}$.

Structure-activity relationship (SAR) studies of quinolone antibacterial agents have revealed that the $\mathrm{C}-7$ position is the most adaptable site for chemical modification and this area greatly influences their potency, spectrum and safety ${ }^{4,5}$. More interestingly, previous work on piperidinyl quinolones suggested the importance of the relative positions of substituent groups on the C-7 side chain with respect to biological activity. For example, 7-(4-alkoxyimino-3-aminopiperidin-yl)quinolones might have completely different activity compared to their position isomers containing a 3-alkoxyimino-4/5-aminopiperidine moiety at the $\mathrm{C}-7$ position ${ }^{6-8}$.

\section{EXPERIMENTAL}

Zabofloxacin (DW224a, Fig. 1), a novel naphthyridone agent containing an oxime-functionalized spirocycle scaffold as the C-7 substituent, showed excellent activity against Grampositive resistant bacteria, associated with very low toxicity and favorable pharmacokinetic profiles ${ }^{9}$. Therefore, we have designed and synthesized a series of novel naphthyridone derivatives having 8-alkoxyimino-1,6-dizaspiro[3.4]octane scaffolds 1-9 (Fig. 1), the position isomers of the side chain at the C-7 position of zabofloxacin ${ }^{10}$. Their in vitro antibacterial activity against representative strains and structure-activity relationship were studied in this paper.

\section{RESULTS AND DISCUSSION}

The target compounds 1-9 were synthesized according to our previous work ${ }^{10}$ and evaluated for their in vitro antibacterial activity against representative Gram-positive and Gram-negative strains using standard techniques ${ }^{11}$. Minimum inhibitory concentration (MIC) is defined as the concentration of the compound required to give complete inhibition of bacterial growth and MICs of the synthesized compounds along with the reference drug levofloxacin (LVFX) for comparison are reported in Table-1. These data suggested that in vitro antibacterial activity of the target compounds was less than the reference against all of the tested Gram-positive and Gramnegative strains with few exceptions. Nevertheless, compounds 1-5 and 7 showed good activity against four strains of 


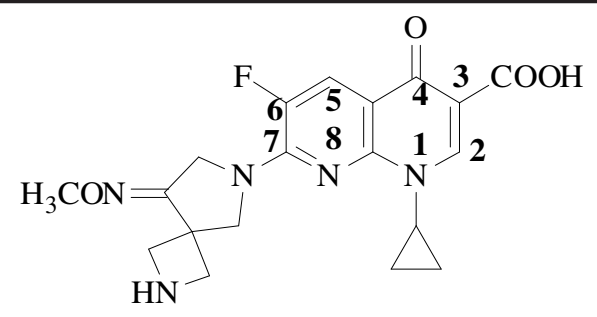

Zabofloxacin<smiles>CCON=C1CN(c2nc3c(cc2F)c(=O)c(C(=O)O)cn3C2CC2)CC12CCN2</smiles><smiles>CCON=C1CN(c2nc3c(cc2F)c(=O)c(C(=O)O)cn3-c2ccc(F)cc2F)CC12CCN2</smiles><smiles>CCON=C1CN(c2nc3c(cc2F)c(=O)c(C(=O)O)cn3C2CC2)CC12CCN2C</smiles>

Fig. 1. Chemical structures of zabofloxacin and its derivatives

methicillin-sensitive Staphylococcus aureus (MSSA) (MICs: $0.25-1 \mu \mathrm{g} / \mathrm{mL}$ ) and two strains of Pseudomonas aeruginosa (MICs: 0.25-0.5 $\mu \mathrm{g} / \mathrm{mL}$ ). Among of them, compounds 3 and 7 (MICs: $0.25 \mu \mathrm{g} / \mathrm{mL}$ ) were found to be 4 times more potent than or comparable to LVFX against $P$. aeruginosa, the important conditioned pathogen on hospital infection.

The structure-activity relationship activity revealed that: (1) for N-1 position with the same C-7 substituent, cyclopropyl > 2,4-difluorobenzyl $\approx$ ethyl; (2) 6-fluoro naphthyridones > 6-hydrogen analogs; (3) introduction of $\mathrm{N}$-methyl group at C7 position decreased their activity against Gram-positive strains ( 2 vs 7), but appeared no effect for their activity against Gramnegative strains.

\section{Conclusion}

In summary, a series of novel naphthyridone derivatives containing 8-alkoxyimino-1,6-dizaspiro[3.4]octane scaffolds were evaluated for their in vitro antibacterial activity against representative strains. Although most of the target compounds were generally less active than the reference against the tested Gram-positive and Gram-negative strains, compounds 1-5 and 7 showed good activity against MSSA and $P$. aeruginosa. Among of them, compounds 3 and 7 (MICs: $0.25 \mu \mathrm{g} / \mathrm{mL}$ ) were found to be 4 times more potent than or comparable to LVFX against $P$. aeruginosa.

\section{ACKNOWLEDGEMENTS}

This work was supported by the National S\&T Major Special Project on Major New Drug Innovation (Number 2012ZX09301002-001-017).

\section{REFERENCES}

1. K. Drlica, M. Malik, R.J. Kerns and X.L. Zhao, Antimicrob. Agents Chemother., 52, 385 (2008).

2. D.J. Dwyer, M.A. Kohanski, B. Hayete and J.J. Collins, Mol. Syst. Biol., 3, 1 (2007). 
TABLE-1

IN VITRO ANTIBACTERIAL ACTIVITY OF COMPOUNDS 1-9 AGAINST GRAM-POSITIVE AND GRAM-NEGATIVE STRAINS

\begin{tabular}{|c|c|c|c|c|c|c|c|c|c|c|}
\hline \multirow{2}{*}{ Strains } & \multicolumn{10}{|c|}{ MIC $(\mu \mathrm{g} / \mathrm{mL})$} \\
\hline & 1 & 2 & 3 & 4 & 5 & 6 & 7 & 8 & 9 & LVFX \\
\hline S.a. & 0.25 & 0.25 & 0.25 & 2 & 0.5 & 0.25 & 0.5 & 2 & 2 & 0.125 \\
\hline MRSA1* & 64 & 32 & 32 & 64 & 128 & 32 & $>128$ & $>128$ & $>128$ & 32 \\
\hline MRSA2* & 64 & 32 & 32 & 128 & 128 & 32 & $>128$ & $>128$ & $>128$ & 32 \\
\hline MRSA3* & 32 & 32 & 32 & 128 & 64 & 32 & $>128$ & $>128$ & $>128$ & 16 \\
\hline MSSA1* & 0.25 & 0.25 & 0.25 & 0.5 & 0.5 & 1 & 0.5 & 4 & 4 & 0.125 \\
\hline MSSA2* & 0.5 & 0.5 & 1 & 0.5 & 0.5 & 4 & 1 & 8 & 8 & 0.5 \\
\hline MSSA3* & 0.5 & 0.25 & 0.25 & 0.5 & 0.5 & 4 & 0.5 & 2 & 2 & 0.25 \\
\hline MRSE1* & 32 & 64 & 16 & 64 & 128 & $>128$ & $>128$ & $>128$ & $>128$ & 8 \\
\hline MRSE2* & 4 & 2 & 8 & 8 & 16 & 128 & 16 & $>128$ & $>128$ & 4 \\
\hline MSSE1* & 64 & 64 & 64 & 64 & 128 & 128 & $>128$ & $>128$ & $>128$ & 128 \\
\hline MSSE2* & 8 & 16 & 16 & 16 & 64 & 128 & 4 & $>128$ & $>128$ & 4 \\
\hline MSSE3* & 8 & 16 & 8 & 16 & 64 & 128 & 4 & $>128$ & $>128$ & 4 \\
\hline MSSE4* & 64 & 64 & 64 & 64 & 128 & 128 & $>128$ & $>128$ & $>128$ & 128 \\
\hline E.fa.1 & 32 & 32 & 8 & 16 & 64 & 128 & $>128$ & 64 & $>128$ & 8 \\
\hline E.f3.1 & 32 & 64 & 32 & 32 & 128 & 128 & $>128$ & 128 & 128 & 16 \\
\hline E.fm.1 & 32 & 64 & 32 & 64 & 64 & 64 & $>128$ & $>128$ & $>128$ & 32 \\
\hline E.fm. 2 & 32 & 2 & 2 & 4 & 8 & 64 & $>128$ & 32 & 64 & 2 \\
\hline E.fm. 3 & 32 & 64 & 32 & 64 & 64 & 64 & $>128$ & $>128$ & $>128$ & 32 \\
\hline S.p. $1^{*}$ & 16 & 16 & 16 & 16 & 8 & 32 & 32 & 32 & 64 & 16 \\
\hline S.p. $2 *$ & 4 & 4 & 8 & 2 & 4 & 32 & 4 & 16 & 64 & 1 \\
\hline S.p. $3^{*}$ & 32 & 32 & 64 & 64 & 64 & 64 & $>128$ & $>128$ & $>128$ & 32 \\
\hline S.p. $4 *$ & 2 & 2 & 1 & 2 & 8 & 32 & 4 & 32 & 32 & 1 \\
\hline S.p. $5^{*}$ & 0.125 & 0.25 & 1 & 2 & 0.5 & 1 & 2 & 16 & 32 & 0.06 \\
\hline E.co. & 0.06 & 0.25 & 0.5 & 1 & 0.5 & 0.5 & 2 & 8 & 16 & 0.008 \\
\hline E.co.1 & $>128$ & $>128$ & $>128$ & $>128$ & $>128$ & $>128$ & $>128$ & $>128$ & $>128$ & 4 \\
\hline E.co. 2 & 16 & 4 & 16 & 16 & 8 & 8 & 16 & 32 & 128 & 0.25 \\
\hline E.co. 3 & 32 & $>128$ & 64 & $>128$ & 128 & 128 & $>128$ & $>128$ & $>128$ & 4 \\
\hline E.co. $2 *$ & $>128$ & $>128$ & $>128$ & $>128$ & $>128$ & $>128$ & $>128$ & $>128$ & $>128$ & 16 \\
\hline E.co. $3^{*}$ & 128 & 128 & $>128$ & $>128$ & $>128$ & $>128$ & $>128$ & $>128$ & $>128$ & 8 \\
\hline K.p.1 & $>128$ & $>128$ & $>128$ & $>128$ & $>128$ & $>128$ & $>128$ & $>128$ & $>128$ & 32 \\
\hline K.p.2 & 16 & 4 & 8 & 16 & 8 & 8 & 16 & 32 & 128 & 1 \\
\hline K.p.4 & $>128$ & $>128$ & $>128$ & $>128$ & $>128$ & $>128$ & $>128$ & $>128$ & $>128$ & 32 \\
\hline K.p. $1 *$ & $>128$ & $>128$ & $>128$ & $>128$ & $>128$ & $>128$ & $>128$ & $>128$ & $>128$ & 4 \\
\hline K.p. $2^{*}$ & $>128$ & $>128$ & $>128$ & $>128$ & $>128$ & $>128$ & $>128$ & $>128$ & $>128$ & 8 \\
\hline K.p. $3 *$ & $>128$ & $>128$ & $>128$ & $>128$ & $>128$ & $>128$ & $>128$ & $>128$ & $>128$ & 8 \\
\hline P.a. ${ }^{*}$ & 0.5 & 0.5 & 0.25 & 0.5 & 0.5 & 2 & 0.25 & 2 & 2 & 0.25 \\
\hline P.a. ${ }^{*}$ & 0.5 & 0.5 & 0.25 & 0.5 & 0.5 & 2 & 0.25 & 2 & 2 & 1 \\
\hline
\end{tabular}

Abbreviations: S.a., Staphylococcus aureus ATCC259223; MRSA1, methicillin-resistant Staphylococcus aureus 10-11; MRSA2, methicillinresistant Staphylococcus aureus 10-13; MRSA3, methicillin-resistant Staphylococcus aureus 10-15; MSSA1, methicillin-sensitive Staphylococcus aureus 10-11; MSSA2, methicillin-sensitive Staphylococcus aureus 10-13; MSSA3, methicillin-sensitive Staphylococcus aureus 10-14; MRSE1, methicillin-resistant Staphylococcus epidermidis 10-10; MRSE2, methicillin-resistant Staphylococcus epidermidis 10-13; MSSE1, methicillinsensitive Staphylococcus epidermidis 10-11; MSSE2, methicillin-sensitive Staphylococcus epidermidis 10-13; MSSE3, methicillin-sensitive Staphylococcus epidermidis 10-14; MSSE4, methicillin-sensitive Staphylococcus epidermidis 10-15; E.fa.1, Enterococcus faecalis 10-5; E.fa.2, Enterococcus faecalis 10-6; E.fa.3, Enterococcus faecalis 10-7; E.fm.1, Enterococcus faecium 10-5; E.fm.2, Enterococcus faecium 10-6; E.fm.3, Enterococcus faecium 10-9; S.p.1, Streptococcus pneumoniae 10-1; S.p.2, Streptococcus pneumoniae 10-2; S.p.3, Streptococcus pneumoniae 10-4; S.p.4, Streptococcus pneumoniae 10-5; S.p.5, Streptococcus pneumoniae 10-6; E.co., Escherichia coli ATCC25922; E.co.1, Escherichia coli 10-1; E.co.2, Escherichia coli 10-2; E.co.3, Escherichia coli 10-3; E.co.4, Escherichia coli 10-4; K.p.1, Klebsiella pneumoniae 10-1; K.p.2, Klebsiella pneumoniae 10-2; K.p.3, Klebsiella pneumoniae 10-3; K.p.4, Klebsiella pneumoniae 10-4; P.a.1, Pseudomonas aeruginosa 10-9; P.a.2, Pseudomonas aeruginosa 10-18; *, extended spectrum beta-lactamases (ESBLs)-producing; LVFX, levofloxacin

3. M.L. Liu and H.Y. Guo, World Notes Antibiot., 27, 69 (2006).

4. A. Bryskier and J.F. Chantot, Drugs, 49(Suppl. 2), 16 (1995).

5. H. Koga, A. Itoh, S. Murayama, S. Suzue and T. Irikura, J. Med. Chem., 23, 1358 (1980).

6. Z. Dang, Y.S. Yang, R.Y. Ji and S.H. Zhang, Bioorg. Med. Chem. Lett., 17, 4523 (2007).

7. X.Y. Wang and Q. Guo, Acta Pharmacol. Sin., 43, 819 (2008).

8. Y.B. Zhang, G.Q. Li, M.L. Liu, X.F. You, L.S. Feng, K. Lv, J. Cao and H.Y. Guo, Bioorg. Med. Chem. Lett., 21, 928 (2011).

9. R.N. Jones, D.J. Biedenbach, P.G. Ambrose and M.A. Wikler, Diagn. Microbiol. Infect. Dis., 62, 110 (2008).
10. L.-S. Feng, M.-L. Liu, S. Wang, Y. Chai, K. Lv, G.-Z. Shan, J. Cao, S.-J. $\mathrm{Li}$ and H.-Y. Guo, Tetrahedron, 67, 8264 (2011).

11. MICs were determined as described by the NCCLS (see: National Committee for Clinical Laboratory Standards, Performance Standards for Antimicrobial Susceptibility Testing: 11th Informational Supplement, vol. 21, National Committee for Clinical Laboratory Standards, Wayne, PA, 2001, M100-S11. The MIC was defined as the lowest concentration of each compound resulting in inhibition of visible growth of bacteria after incubation at $35{ }^{\circ} \mathrm{C}$ for $18 \mathrm{~h}$. 